\title{
Analysis on the Causes and Countermeasures of Expatriate Management Problems in Multinational Corporations
}

\author{
Yahang Tong*
}

Hebei University of Economics and Business, Shijiazhuang, China

*Corresponding author. Email: 1371936397@qq.com

\begin{abstract}
With the continuous development of economic globalization, the trade between countries is becoming more and more frequent, and the economic exchanges between countries are also increasing, which also promotes the emergence of more and more transnational enterprises. Human resource management in enterprises is a very important factor affecting the competitiveness of multinational enterprises, among which the management of expatriates is a great challenge for the managers of multinational companies. Therefore, it is necessary to analyse the problems encountered in the management of expatriates and summarize the countermeasures, which will provide the managers of multinational companies with good coping strategies for the expatriate problems, so as to promote the good operation of the company.

This paper uses related theories and cases to analyse the problem of expatriate management in multinational corporations. Combining the theory of human resource configuration, Hofstede cultural dimensions theory and Maslow's hierarchy of needs theory, find out the expatriates in the process of sending the problem, and once again according to the related theory put forward relevant countermeasures to solve these problems, to improve the management level of foreign multinational company sent personnel, makes the expats can complete the task on schedule.
\end{abstract}

Keywords: Transnational corporations, Expatriate personnel, Management.

\section{跨国公司外派人员管理问题成因分析及对策研究}

\author{
佟亚杭*
}

河北经贸大学, 石家庄，中国

*通讯作者.邮箱: 1371936397@qq.com

\section{中文摘要}

随着经济全球化的不断发展, 各国之间的贸易往来越来越频繁, 国家与国家之间的经济交流也越来越多, 这也 促进了越来越多跨国企业的产生。而企业中的人力资源管理部分是影响跨国企业竞争力的一个很重要的因素, 其中外派人员的管理问题对于跨国公司的管理者来说是很大的挑战。因此对于外派人员管理中所遇到的问题的 分析及应对的策略的总结是很有必要的, 这会给跨国公司的管理者针对外派人员问题提供良好的应对策略, 从 而促进公司的良好运营。 
本文运用相关理论与案例来分析跨国公司外派人员管理问题。结合人力资源配置理论、霍夫斯泰德文化维度理 论和马斯洛需求层次理论等理论, 找出外派人员在派遣过程中所遇到的问题, 并再次根据相关理论提出相关的 对策解决这些问题，来提高跨国公司对外派人员的管理水平，使得外派人员可以如期完成任务。

关键词：跨国公司，外派人员，管理

\section{1. 绪论}

\section{1. 研究背景}

随着全球各国之间的经济联系越来越紧密, 相互 之间的贸易往来越来越频繁, 经济组织结构也产生了 新的变化, 跨国公司越来越多。随之而来, 这些跨国 公司就会产生新的问题——跨国公司外派人员的管 理问题。对外派人员进行科学有效的管理, 促使他们 可以顺利完成跨国公司委派给他们的海外派遣任务, 这对于跨国公司的经营能否成功来说是至关重要的。

从历史角度来看, 跨国公司的出现开始于二战以 后, 伴随着第三次科技革命的开始, 经济全球一体化 的发展开始进入了下一个阶段中，而战后世界全球经 济活动主要崛起的一个基本特征则是跨国公司的崛 起。二战后的第三次科技革命关于经济方面则是对于 国际商业经营模式进行革命, 因此最终导致世界经济 的跨国化, 从而致使越来越多的跨国公司的出现。然 而, 虽然跨国公司的发展速度很快, 但是却面临着派 遣外派人员不成功的问题。这对于企业来说, 不仅要 承担着关于对外派人员投资的这些人力资本的费用, 而且还可能危及与东道国的关系。而对于个人来说, 则会直接面临未来的职业发展的难题。

\section{2. 研究意义}

虽然目前有越来越多的跨国公司，但是大多数的 跨国公司外派效果并没有达到所预期的那样, 普遍存 在着外派失败率较高、外派人员绩效低和归国人员的 流失率较高等与跨国公司外派人员管理问题有关。具 体来说, 他们可能会因自己的能力不足达不到所胜任 工作需要的能力, 也可能会因无法适应国外的生活而 提前回国, 未能如期完成工作任务, 也会出现虽然完 成工作, 但是未能达到所预期的工作绩效目标, 甚至 还可能会出现在外派人员在任期结束后, 并没有回到 原来的公司里, 反而会进入竞争对手的公司工作的情 况等。因此, 通过研究跨国公司外派人员管理问题, 从而找到相应的解决方案, 对于一个跨国企业是非常 有必要的。这样可以帮助跨国企业深刻的来认识如何 去科学有效的派遣外派人员, 提高派遣外派人员的成 功率, 进一步提高跨国企业管理外派人员的能力, 从
而不仅能避免跨国企业因派遣失败所带来的经济而 产生危急的情况的不良后果, 同时对于外派人员个人 的未来的职业发展也有很多有利的地方。

\section{3. 论文的框架和思路}

本文以跨国公司外派人员作为研究对象, 采用理 论分析与个案实践相结合的方法, 首先对跨国公司、 外派、外派人员、人力资源配置理论、霍夫斯泰德文 化维度和马斯洛需求层次理论等概念进行了界定。接 着分析外派人员管理所存在的问题，如：外派人员选 拔不合理、外派人员的培训内容不充分、缺乏对外派 人员的考核机制、缺乏对外派人员的回任管理、忽略 了外派人员家庭的影响等问题进行了分析, 随后, 引 入了两个案例, 一个是美国跨国公司外派人员管理, 另一个是中国跨国公司外派人员管理。最后, 在分析 了美国跨国公司外派人员管理和中国跨国公司外派 人员管理的存在的问题之后, 总结了针对跨国公司外 派人员管理问题提出了相应的一些建议。

\section{2. 相关理论综述}

\section{1. 基本概念}

\subsection{1. 跨国公司的定义}

跨国公司是在本国设立母公司, 对其他国家进行 投资来设立其子公司。各个子公司会受到母公司的影 响, 并且接受母公司指令来进行其经济活动。

跨国公司崛起的根本原因在于新中国成立 70 年特 别是改革开放 40 多年以来中国经济的持续发展为中 国跨国公司的崛起创造了雄厚的国内经济基础和广 阔的国际市场环境, 其对国际社会科技发展和人类文 明进步的积极影响广泛、系统而深远[1]。

\subsection{2. 外派的定义}

外派是指跨国公司的总部派遣母国工作人员或第 三国工作人员到国外的子公司或分公司在预定的时 间段内工作, 达到任期之后, 再返回原来的母公司继 续工作。外派的时间大部分在 6 个月到 5 年之间, 而 
如果外派时间比较短，则可以被理解成工作人员去是 因为要完成工作而出差, 如果外派时间很长的话, 则 理解为是去到国外定居。

\subsection{3. 外派人员的定义}

外派人员主要指由母公司委派到其子公司的人员, 而外派到子公司的人员大都是那些能力很强的精英 人员, 他们通常是为子公司企业提供组织需要的可转 移的知识、技能和经验。他们也是承担为使母公司与 子公司的管理模式与工作标准一致的责任, 提高子公 司的经营管理水平与能力。

\section{2. 相关理论}

\subsection{1. 人力资源配置理论}

在企业中, 为了提高企业的经营能力, 提高员工 的工作积极性与工作效率, 人力资源配置理论要求管 理者可以根据每一位员工的能力与爱好, 在尊重员工 的个人意愿的前提下, 按照他们的能力的层级, 把员 工安排到与他们的能力最匹配的岗位上, 让员工在他 们的岗位上发挥出他们最大的能力, 从而发挥出人力 资源的最大作用。

\subsection{2. 霍夫斯泰德文化维度理论}

霍夫斯泰德文化维度理论是荷兰心理学家吉尔 特·霍夫斯泰德提出，此理论可以通过不同的维度将 人群加以区分。企业管理者可以根据此理论理解不同 群体的差异, 从而可以更好的实现人员的管理。以下 是用中国与美国的霍夫斯泰德文化维度差异来做霍 夫斯泰德文化维度分析。

（一）权力距离是处于权力比较弱势地位的员工在 机构和组织中能够接受这种分配不平等的程度。美国 的权力距离指数较低, 上级对员工的约束较小。而中 国等亚洲国家由于体制的关系, 上级对员工的约束较 大，员工对上级甚至带有畏惧感。

(二) 不确定性规避是指当人们遇到未来不可知的 事情时所做出的反应, 是勇敢尝试迎难而上还是选择 退缩逃避。美国是不确定性回避指数比较低的国家, 他们在遇到这些不确定的事情会选择直面它并去尝 试; 而中国的不确定性回避指数比较高, 他们会尽量 避免没有把握的事情发生。

（三）个人主义/集体主义维度是衡量人们只顾及自 己还是更考虑周围集体的利益的维度。欧美企业员工 更重视自己的个人利益, 对于是否能融入集体并不是 很重视; 而中国文化强调群体至上, 中国企业员工更 注重和周围同事及领导的关系并避免发生冲突。

(四) 男性化与女性化维度主要衡量人们是更加看 重通过竞争的方式成就自己还是讲究和谐, 更加关心
他人和提高生活质量方面。中国和美国都是以成功为 导向, 充满干劲, 男性化数值较大。

(五) 长期取向与短期取向维度是看这个国家的人 们更愿意保持过去的状态，还是会更加务实，灵活的 为当前的环境做准备。中国的得分较高, 这意味着它 是一个非常务实的文化。他们有能力使传统适应变化 的环境, 有强烈的储蓄和投资倾向, 节俭, 以及在取 得结果时的毅力。美国得分较低, 美国企业以短期为 基础衡量业绩，损益表按季度发布。

(六) 自身放纵与约束维度指的人们控制自己内心 欲望的程度。中国的得分较低, 中国是一个克制的社 会, 中国社会的人认为他们的行为受到社会规范的约 束, 认为放纵自己是不对的。而美国得分较高, 美国 也被认为是一个宽容的社会。

\subsection{3. 马斯洛需求层次理论}

亚伯拉罕·哈罗德·马斯洛于 1943 年初次提出了“需 要层次”理论, 他把人类纷繁复杂的需要分为八个层 次。

生理的需要: 人们需要食物、水分和空气等维持 生命最基本的物质。

安全需要:人们需要一个可以给他们带来稳定和安 全的地方, 可以让他们能减少恐惧和焦虑。

归属和爱的需要：人们需要与其他人建立感情上 的联系, 来获得归属感和情感的需求。

尊重的需要:需要别人尊重自己的尊严和自己所获 得的成就, 还有对别人的尊重。

认知需求: 想获得知识, 来去理解未知的事情, 满足自己的好奇心。

审美需求: 人们需要欣赏周围的美的事物。

自我实现的需要:人们想提高自己的能力, 以便可 以实现自己所追求的理想。

超越需要: 人对不断超越自己有需求。

\section{3. 外派人员管理所存在的问题}

\section{1. 外派人员选拔不合理}

在多数跨国企业中，非常需要一些国际化经营人 才加入本跨国企业，因此企业的人力资源管理部门在 外部招聘选拔这些国际化经营人才的时候, 会因缺乏 统一的标准导致选拔的人才质量良莠不齐[2]。而在企 业内部选拔时，同样会因没有统一科学的标准，而可 能出现选拔高层管理者时 “任人唯亲”的现象，比如一 些跨国企业的关键岗位一一总经理和财务总监等把 握公司命脉的核心岗位任命自己的亲戚, 并没有按照 公平与公正的选拔制度来选拔，而那些通过关系在关 键岗位上岗的人员，他们可能会因为缺乏管理经验而 给公司带来不可预料的严重后果。而在选拔中低层管 
理者时，管理者本身没有遵循人力资源配置理论，只 关注工作本身这唯一选拔的目的, 管理者没有跟据员 工的专业特长及工作爱好将员工安置到最有利于他 们发挥其优势的岗位上, 因此违背了这些工作人员的 初衷。

\section{2. 外派人员的培训内容不充分}

当外派人员刚到海外的子公司或分公司工作的时 候, 很有可能因自身与当地的工作同事的存在文化上 的差异，外派人员在东道国的环境中会面临不同文化 之间的一些问题，甚至会导致冲突。用霍夫斯泰德文 化维度理论分析, 不同国家之间六个维度方面存在差 异, 可能会因此导致外派人员和当地的工作伙伴处理 事情的思维和方式不一样[3]。文化差异性可能给合资 企业带来沟通冲突, 甚至可能影响企业之间的合作, 因此在工作上可能会出现分析一些分歧。这不仅会影 响外派人员与所属子公司或分公司的工作人员或者 管理者的相处的关系, 严重的话, 还会因为工作上的 严重分析导致外派人员最终无法达到任务完成所预 期的效果, 而且可能会出现提前离职的现象, 最终导 致外派失败。而这都是由于管理者对于外派人员的培 训内容不充分所造成的。

\section{3. 缺乏对外派人员的绩效考核机制}

一些跨国公司为了节约人力资本，往往让企业中 的外派人员兼任多个职位, 而且有的企业缺乏专业性 的绩效考核制度和绩效考核标准, 考核时全凭借高层 管理层领导者自己的主观判断, 因此可能会出现对于 这些外派人员的考核造成不公平的现象 [4]。用马斯洛 需求层次理论来解释, 这样考核的不公平会违背人对 于尊重的需要, 会让外派人员觉得自己所做出的成果 不被尊重, 轻而易举的就被抹杀, 也有可能是考核目 标过于单一, 并不全面。这些都会导致外派人员认为 考核缺乏人性化和科学性, 因此, 也会降低外派人员 的工作的积极性, 最终导致无法如期完成外派任务, 导致外派失败。

\section{4. 缺乏对外派人员的回任管理}

因为外派人员长期在国外工作, 因离开国内很长 时间, 周围的以前同事很有可能换成了全新的不熟悉 的人。并且, 公司可能对于这些归国的外派人员的工 作安排不合理, 可能会让这些归国的外派人员去处理 解决和当时他们去海外联系不紧密的工作, 让他们在 海外工作所学到的那些工作经验和技能没有在他们 现在的工作中派上用场[5]。根据马斯洛需求层次理论 中的人们对于自我实现的需要和归属和爱的需要理 论, 外派人员想和同事建立亲密的关系, 并且想让自 己在海外学到的知识排上用场, 以变更好的实现自己 的价值。因此他们会觉得公司的管理者没有肯定他们 在海外工作所学到的经验与技能, 并且和周围同事相
处没有以前那么自然亲密, 以至于他们的工作积极性 下降, 甚至他们会有离职的现象。

\section{5. 忽略外派人员家庭的影响}

外派人员在工作与家庭角色之间的调节非常重要, 家庭因素是影响外派人员能否外派成功的重要因素 之一[6]。由于外派人员去子公司或分公司的任期一般 都比较长, 因此可能会导致这些外派人员与其配偶和 孩子长时间缺乏紧密的联系, 缺乏有效的沟通, 从而 导致家庭成员关系之间的感情变得很淡, 可能会存在 争吵, 因而使外派人员会影响工作，无法专心的投入 到工作之中, 造成最终完成既定的工作任务, 导致外 派失败[7]。根据马斯洛需求层次理论中的人们对于爱 的需要理论, 外派人员的家庭是外派人员的坚强并重 要的后盾, 外派人员有和家人和睦相处的需求, 如果 公司未能安抚好这些外派人员的家人,那么外派人员 家庭之间会因此产生矛盾，因此这会严重打击外派人 员的工作的积极性, 进而出现外派人员无法最终完成 既定的工作任务，导致外派失败的严重后果。

\section{4. 美国跨国公司外派人员管理的实践及其经 历}

\section{1. 案例背景}

戴尔是一家总部位于美国德克萨斯州朗德罗克的 世界五百强企业, 由迈克尔·戴尔于 1984 年创立。戴 尔以生产、设计、销售家用以及办公室电脑而闻名, 不过它同时也涉足高端电脑市场, 生产与销售服务器、 数据储存设备、网络设备等。戴尔公司一直名列《财 富》杂志评选的“最受仰慕的公司”。戴尔公司经营理 念是, 无论普通人、机构, 还是社区, 只要使用合适 的工具, 掌握一定的技能, 一切皆有可能。但是其作 为一家跨国公司, 在管理外派人员时会出现违背员工 意愿、对外派人员培训内容不充分和对外派人员的考 核机制不合理等问题。

\section{2. 出现的问题及原因}

\subsection{1. 违背员工的意愿}

戴尔公司仅仅以成本和业绩为导向的人力资源战 略, 只关注工作本身这唯一选拔的目的, 虽然可以让 公司快速发展, 可以适应复杂多样的市场环境, 并且 可以在复杂而多样竞争的市场环境中取胜, 但是根据 人力资源配置理论, 管理者只关注工作本身这唯一选 拔的目的, 没有根据员工的自身兴趣和优势来分配工 作, 导致让员工可能长时间做着自己不擅长并内心不 感兴趣的工作，这会大大加重员工的工作负担。很多 戴尔公司的外派人员就因长时间的工作负担, 不仅打 消了工作积极性, 没有如期完成外派任务, 甚至因为 工作负担较重, 倒在了所在的工作岗位上面。 


\subsection{2. 对外派人员培训内容不充分}

因公司未能及时意识到文化差异，比如戴尔公司 的管理风格是强大的销售压力, 非常缺乏人性的关怀。 在美国, 美国员工可以接受紧张的劳动状态、工作模 式和压力。而在中国, 中国员工不能接受紧张的工作 模式, 并且严重的工作压力会更让中国员工产生不适 应。用霍夫斯泰德文化维度理论解释, 从个人主义和 集体主义维度考虑, 美国重视个人的利益, 不太重视 自己是否和周围人相处, 因此, 当从美国母公司委派 一名工作人员来管理建立在中国的分公司的话, 这名 美国国籍的外派人员只强调自己如何把任务做好, 因 此在实行制度时, 没有太顾及其他员工的感受, 只强 调达到最终任务的目标。因此实行这些制度对于中国 员工不太合理的制度时, 就会造成中国员工从心底的 排斥感, 降低中国员工的工作积极性, 最终还是会导 致外派人员无法最终完成既定的工作任务, 最终导致 外派失败的严重后果。

\subsection{3. 对外派人员的考核机制不合理}

戴尔公司的企业文化是以“业绩”，公司总裁只关 心营业毛利, 因此, 受这种企业文化影响, 人力资源 管理模式也是以“绩效”作为导向的。因此公司在对于 外派人员的考核标准中也是以“绩效”为唯一导向, 只 关注外派人员的“绩效”, 这给外派人员带来了很多心 理上的压力, 打击外派人员的工作积极性。并且, 以 “绩效”作为唯一的标准考核外派人员也是不公平的 一种表现, 考核目标过于单一, 并不全面而导致外派 人员认为考核缺乏人性化和科学性[8]。用马斯洛需求 层次理论中的尊重的需求层次解释, 人们有别人对自 己能力认可以获得别人尊重的需求, 但只对单一目标 的考核显然是让很多外派人员无法获得别人对他们 自己成绩的认可。因此, 外派绩效维度不应仅仅局限 于工作任务的最终的完成度, 还应包括衡量工作态度 方面的指标, 如外派人员工作对这次外派的满意度和 自身是否愿意提早回国意向等 [9], 否则, 会降低外派 人员的工作的积极性, 最终导致无法如期完成外派任 务, 导致外派失败。

\section{5. 中国跨国公司外派人员管理的实践及其经} 历

\section{1. 案例背景}

中国远洋运输公司是全球规模比较大的海洋运输 公司之一, 隶属于国有企业。该公司成立于 1961, 入 选为《财富》世界 500 强企业。作为一家中国的跨国 公司, 中远集团不光逐渐在在航运、物流和修造船领 域方面提高自己的实力, 还积极建立与其客户、员工 和合作伙伴的关系, 尽最大所能为股东和社会创造财 富。但是其作为一家跨国公司, 在管理外派人员时会 出现缺乏对外派人员的回任管理和忽略了外派人员 家庭的影响等问题。

\section{2. 出现的问题及原因}

\subsection{1. 缺乏对外派人员的回任管理}

如果员工出色地完成了外派任务, 但在归国后即 离职, 整体上来看仍然是企业的较大损失[10]。因此, 需要加强对外派员工归国后的研究, 进而帮助企业采 取措施留住具有成功外派经验的员工当外派人员任 期回国后, 他们会发现公司的工作环境和生活环境都 会有很大的变化。因此, 他们可能会对当前的工作环 境有些不适应。比如对于人事变动方面, 可能过去他 们的部门的直属领导或者部门同事已经被更替掉了, 也有可能会被安排到另一个不同于原来的部门里去 工作, 而且安排的工作可能也与海外所学到的工作经 验和技能并没有很大关联, 因此在海外所学到的东西 没有在工作上得到应用。根据马斯洛需求层次理论中, 人们对于归属和爱的需求和自我实现的需求解释, 外 派人员到一个全新的环境, 和周围人是陌生的感情, 让外派人员没有归属感, 而且又不能把从海外所学的 新知识应用到工作中, 这会让外派人员感觉缺少了价 值, 因此, 会打消外派人员工作积极性, 导致最终外 派失败。

\subsection{2. 忽略了外派人员家庭的影响}

家庭因素对于外派人员能否最终可以如期完成外 派任务已成为一个十分重要且不可忽略的因素, 其中 $65 \%$ 的外派人员有配偶, 而在有配偶的家庭中, 其中 有 $67 \%$ 的家庭都有孩子, 因此配偶的工作和他们子女 的教育问题在很大的程度中对于外派人员的工作效 率和他们在工作中所投入的精力产生十分重要的影 响。家人是外派人员坚强的后盾,公司安抚好员工的家 人,也是在激励外派人员努力工作 $[11]$ 。根据马斯洛需 求层次理论, 人们对于归属和爱有需求, 如果一旦处 理不好外派人员的配偶的工作和他们子女的教育问 题, 可能影响家庭成员内部之间的关系, 因此会降低 外派人员的工作积极性, 影响外派人员最终的业绩, 最终导致无法如期完成外派任务, 导致外派失败。

\section{6. 外派人员管理问题应对策略}

\section{1. 提高外派人员的选拔标准}

根据人力资源配置理论，管理者应该要根据外派 人员的能力和特长爱好, 来安排外派人员到适合的岗 位上。选拔应聘者首先要以工作岗位的具体要求建立 一套科学的选拔标准, 比如要有很强的语言能力 (委 派到第三国家的语言能力）、较强的沟通能力、对该 岗位的相关工作都很多的工作经验等, 如果是委派到 第三国分公司去担任一些技术方面的岗位比如工程 师的话, 还需要丰富的实践经验, 并且要对涉及到的 所有操作步骤都了然于胸, 方便指导当地的技术工人 [12]。这样可以避免“任人唯亲”的不公平现象的出现。 另外, 选拔过程中也要考虑到外派人员的心理因素和 
身体因素的影响，考虑到外派人员的具体身体情况与 外派人员的心中的意愿, 不可强迫外派人员去某个岗 位任职。

\section{2. 完善外派人员的培训内容}

根据霍夫斯泰德文化维度理论，国家和国家之间 在权力距离、不确定性规避、个人主义集体主义、男 性化女性化、长期取向和短期取向和自身放纵与约束 方面不同，两个国家的管理者和工作人员在处理工作 时会有不同的思维模式和手段，正因为这样，外派人 员和当地公司的工作人员或管理者会产生分歧，导致 外派失败, 因此, 需要对外派人员进行关于文化培训。 比如向外派人员介绍当地国家的基本情况如文化、环 境、当地人的生活习惯、与当地人相处时需要注意的 事项以及人际交往技巧, 还有当地的求助渠道等[13]。 让外派人员提前了解东道国的文化、语言及子公司的 经营管理的模式, 提高外派人员对于所去的分公司的 了解程度, 帮助外派人员适应当地生活和工作, 减少 工作上的摩擦, 提高外派人员工作的积极性, 保证外 派人员如期完成外派任务。

\section{3. 建立规范和公正的外派人员绩效评估}

对外派人员进行科学和合理的绩效评估, 有助于 让外派人员尽快找到差距, 调整工作方式, 更好的去 完成外派任务, 而公司工作人员的工作能力和工作效 率的提升, 也会促进公司的发展。根据马斯洛需求层 次理论, 人们有对别人尊重自己的成绩的需求, 因此 要建立平等合理的考核制度肯定外派人员的成绩, 满 足外派人员的需求。因此为了保证绩效评估的科学和 合理性, 应该确保绩效考核的任务是对员工进行科学 的考核, 根据岗位级别、岗位特点还有业务类型, 并 且结合子公司的经营状况进行考核, 并且为了保证考 核的公正性, 要让外派人员的直属领导、身边的同事、 客户还有母公司的人力资源管理部门进行考核评价。 而且还要建立部门的申诉机制, 如果外派人员感觉考 核不公平, 可立即向直属领导或母公司的人力资源管 理部门进行申诉。

\section{4. 规划好外派人员的回任管理}

根据马斯洛需求层次理论, 人们有对归属和爱的 需求和自我实现的需求。外派人员长期在国外工作, 回国后, 在人事变动方面, 可能会面临新的不熟悉的 同事, 工作方面, 可能会被安排到与之前外派时不相 关的工作, 让外派人员无法将从海外学到的知识用到 新工作中, 因此, 首先对他们进行回国后的培训, 帮 他们努力适应新的环境, 适应新的工作伙伴, 帮助他 们快速适应回国后的工作和生活[14]。再者, 应该给 他们多分配一些相关海外公司的工作, 并且提升他们 在母公司的薪酬水平, 让他们觉得自己在海外工作所 获得的经验和技能是很有用的, 可以激发他们的工作
积极性, 这些都可以满足他们对归属和自我实现的需 求。

\section{5. 加强外派期间的支持和交流}

根据马斯洛需求层次理论, 人们对归属和爱有很 大需求。外派人员的家人对于外派人员能否积极工作 产生重要的影响。如果外派人员与其家人之间产生了 矛盾, 导致外派人员无法从会家庭中满足对于爱和归 属感的需求, 这可能会使外派人员无法像之前一样全 身心的投入到外派工作中, 因此导致外派失败。因此, 企业应该加强对外派人员的家庭方面的重视[15]。首 先, 公司管理人员应该对外派人员及其配偶和子女展 开出国前的培训工作, 提前学习东道国的生文化及生 活习惯, 让其配偶和子女尽快适应国外的生活并且可 以给其配偶提供一些工作岗位, 让其安定工作, 这也 可以促进外派人员更加安心工作。其次, 解决其孩子 的教育问题, 让外派人员带领其子女参加当地学校组 织的活动, 促进孩子与当地孩子的交往, 让孩子尽快 适应国外的教育。最终减少家庭的矛盾, 让外派人员 积极工作, 完成既定的外派任务。

\section{7. 总结}

跨国企业在发展的过程中，外派人员的管理问题 对于一家跨国企业的外派人员能否成功完成外派任 务来说是影响非常大的, 而外派人员能否顺利完成外 派任务对于企业的发展来说也是至关重要的。本文通 过探究外派人员管理中存在的问题导致跨国企业外 派失败的原因, 并且通过实际的案例来研究一些跨国 企业中管理外派人员所产生的问题及原因, 最终来提 出一些解决方案来解决实际中可能会出现的管理外 派人员的问题。这也是给跨国企业在管理外派人员所 提出的一些建议, 从而让跨国企业避免导致外派失败 的不必要的一些问题。

\section{REFERENCES}

[1] Jianyun Bao. The rise of Chinese multinationals, Huawei's Model and the Evolution of the World Pattern [J]. People's BBS,2019(34):12-15.

[2] Baofu Yang. Problems and countermeasures in the management of expatriates in small and medium-sized enterprises [J]. Enterprise reform and management,2020(22):78-79.

[3] Yannan Wang, Zijie li, Banruo Zhang. How to solve the dual pressure of the system in the management of expatriate employees [J]. Hubei Social Sciences,2020(06):60-67.

[4] Qi Wang. Discussion on the problems and countermeasures of performance management of expatriates in multinational corporations [J]. Modern business,2020(32):66-68. 
[5] Xiaoqian Ye, Zequn Wang, Ling Li. Organize career management, The moderating effect of insider identity perception and returned knowledge transfer: individual-organizational consistency matching $[\mathrm{J}]$. Nankai Management Review,2020,23(04):154-165.

[6] Qiuleng Gao, Xinli Huang, Zijie Li, Xiaolin Zhuang. Review and prospect of research on assignment management $[\mathrm{J}]$. Technical and economic,2021,40(01):59-69.

[7] Beiting He, Ran An. Why do you persist under high pressure?The work of Chinese expatriates -- a study on the mechanism of family balance [J]. Nankai Management Review,2020,23(03):141-154.

[8] Xijin Li. Study on performance management countermeasures of expatriates in multinational corporations $[\mathrm{J}]$. Journal of Yellow River Conservancy Technical College,2021,33(01):102-104.

[9] Hui Li, Qun Wang. A study on the influence of cultural intelligence of expatriates on their overseas assignment performance -- based on an empirical study of Chinese "going global" enterprises [J]. To predict,2018,37(02):1-8.

[10] Liang Wang, Xiongying Niu. Review and prospect of studies on expatriate adaptation [J]. East China Economic Management,2018,32(02):176-184.

[11] Qijing Ma. Reasons for the failure of enterprise assignment and the measures to avoid it [J]. Human resource development,2020(14):63-64.

[12] Tianyao Liu, Gengtao Li. Study on management problems and countermeasures of expatriate employees in "going global" enterprises [J]. Management Informatization in China,2019,22(17):103-106.

[13] Lingfeng Yi, Siting Liu, Jie Song, Teng Li. Employee cultural value orientation, intercultural interaction ability and innovation performance: An empirical study of multinational R\&D firms in Shanghai [J]. Journal of East China Normal University (Philosophy and Social Sciences),2021,53(01):155-168+174.

[14] Ling Luo, Juanru Wang, Jie Yang. The influence of vocational competence development on the creativity of returning employees in multinational corporations [J]. Science and Technology Management Research,2019,39(16):167-174.

[15] Quyen T. Dang,Hussain G. Rammal. Japanese expatriates' management in global assignments: A review and research agenda[J]. Thunderbird International Business Review,2020,62(6). 